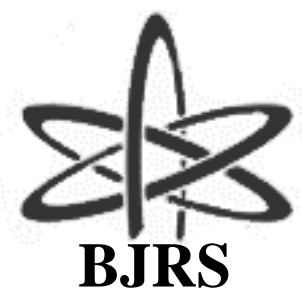

BRAZILIAN JOURNAL

$\mathrm{OF}$

RADIATION SCIENCES

08-02 (2020) 01-13

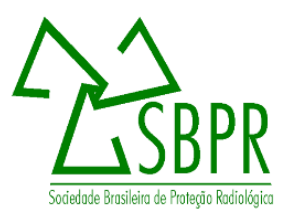

\title{
Validação do controle de qualidade em radiocirurgia utilizando folhas detectoras OSL baseadas na fluorita brasileira
}

\author{
Torquato $^{\mathrm{a}, \mathrm{b}}$ M., Soboll ${ }^{\mathrm{a}}$ D., Pagotto ${ }^{\mathrm{a}}$ I., Horst ${ }^{\mathrm{b}}$ I., Umisedo ${ }^{\mathrm{c}}$ N., Yoshimura $^{\mathrm{c}}$ E., \\ Malthez $^{\text {a A. L. M. C. }}$ \\ ${ }^{a}$ Universidade Tecnológica Federal do Paraná, CEP: 80230-901, Curitiba - PR, Brasil.

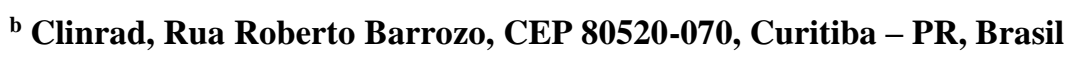 \\ c Universidade de São Paulo, CEP 05508-090 Cidade Universitária, São Paulo - SP, Brasil \\ michele.torquatom@gmail.com
}

\section{RESUMO}

Este trabalho descreve o uso de folhas detectoras OSL de $\mathrm{CaF}_{2}$ em comparação com câmara de ionização para validação da dose planejada em tratamentos de radiocirurgia utilizando a técnica de arcotomia volumétrica modulada (AVM). O controle de qualidade utilizando câmara de ionização foi realizado com uma câmara de ionização PTW semi-flex posicionada no centro de um fantoma com densidade equivalente à da água, como usualmente realizado na verificação da entrega de dose. Para o controle de qualidade utilizando a folha detectora OSL, foram utilizados 3 detectores OSL de $\mathrm{CaF}_{2}$, fitas de $2 \mathrm{~cm}$ de altura e $0,5 \mathrm{~cm}$ de comprimento, envoltos separadamente em plástico pretoradiotransparente, posicionados semelhantemente a câmara de ionização no simulador para verificar as doses de 300, 600 e 2100 cGy planejadas. As fitas foram lidas em um leitor linear OSL no modo pulsado. Os valores de dose avaliados ao longo do comprimento das tiras OSL são compatíveis com os valores fornecidos pelo equipamento e medidos com a câmara de ionização. Além disso, durante o controle de qualidade da câmara de ionização, o equipamento forneceu valores máximos compatíveis com os valores medidos ao longo das fitas detectoras de $\mathrm{CaF}_{2}$. Os resultados mostraram a aplicação de folhas detectoras OSL no controle de qualidade para pequenos tamanhos de campo em radiocirurgia.

Palavras-chave: Controle de qualidade, Radiocirurgia, Detectores OSL em folha. 


\begin{abstract}
This paper reports the use of $\mathrm{OSL} \mathrm{CaF}_{2}$ detector sheets compared to ionization chamber for dose validation planned in radiosurgery treatments using the modulated volumetric arcotherapy technique (VMAT). Quality assurance using an ionization chamber was performed with a semi-flex PTW ionization chamber positioned in the center of a phantom with water equivalent density as usually performed in the check of dose delivery. For quality control using OSL detector sheet, 3 detector the $\mathrm{CaF}_{2} \mathrm{OSL}, 2 \mathrm{~cm}$ high and $0.5 \mathrm{~cm}$ long tapes were used, separately wrapped in radiolucent black plastic and positioned in the position of the ionization chamber in the phantom to verify the doses of 300,600 , and 2100 cGy planned. The detector strips were read on the linear OSL reader in pulsed mode. The dose values evaluated for each of the three schedules along the length of the OSL detector strips are compatible with the values provided by the equipment and measured with the ionization chamber. In addition, during the ionization chamber quality control, the equipment provided maximum values compatible with the values measured along the $\mathrm{CaF}_{2}$ OSL tapes. The results showed the application of OSL detector sheets in quality control for small field sizes in radiosurgery.
\end{abstract}

Keywords: quality assurance, radiosurgery, OSL detector sheet. 


\section{INTRODUÇÃ̃}

Dentre as melhores práticas disponíveis, de acordo com a Organização Mundial de Saúde (OMS), a radioterapia é uma das principais opções no controle do câncer, já que aproximadamente $52 \%$ dos pacientes oncológicos devem receber radioterapia pelo menos uma vez ao longo do tratamento. Ainda quando associada a outras modalidades, a radioterapia tem papel fundamental no tratamento, estimando-se que cerca de $40 \%$ dos pacientes que estão curados do seu câncer passaram por esse tipo de terapia. Além disso, esta modalidade é eficaz nos tratamentos paliativos e no controle de sintomas em casos avançados ou de recidiva [1].

Entre todas as modalidades terapêuticas na radioterapia, a radiocirurgia ganhou destaque como forma efetiva de tratamento associada com baixa morbidade e mortalidade [2]. Em especial, a tecnologia Arcoterapia Volumétrica Modulada (AVM) fornece cobertura ao volume de tratamento preservando os órgãos de risco, com a vantagem da entrega rápida da dose, permitindo mais conforto e reduzindo o movimento durante a execução do tratamento [3].

Nas diversas áreas que utilizam radiação ionizante, devido à proteção radiológica, se faz necessário o controle de qualidade para se conhecer a dose entregue em cada procedimento. Os controles de qualidade e as medidas de dose podem ser feitas através de diversos modos, utilizando equipamentos como câmara de ionização, filme radiocrômico ou detectores luminescentes [4,5].

Baseada nas recomendações internacionais, a Comissão Nacional de Energia Nuclear (CNEN), órgão brasileiro que regulamenta e fiscaliza no Brasil as instituições que utilizam radiação ionizante para fins terapêuticos, não descreve os processos de controle de qualidade, porém estabelece como obrigatório os testes para garantir a segurança nos tratamentos e na precisão para a entrega da dose prescrita [6].

A verificação da dose entregue pelo acelerador linear é feita através do Protocolo TRS 398, o qual foi criado com o objetivo de facilitar a determinação da dose absoluta com exatidão em radioterapia e permitindo o uso de vários dosímetros fornecendo os melhores fatores de correção. A eficiência mecânica nos aceleradores é feita através dos testes apresentados no TECDOC 1151 Aspectos Físicos da Garantia da Qualidade em Radioterapia, os quais visam uma padronização dos parâmetros básicos dos tratamentos radioterápicos [6-8]. 
Nos tratamentos que envolvem alta tecnologia como radioterapia de intensidade modulada (IMRT), radiocirurgia e terapia volumétrica, além dos requisitos mencionados anteriormente, são necessários outros meios de controle de qualidade para garantir a entrega da dose planejada ao paciente. Vale citar que o controle de qualidade é personalizado ao planejamento de cada paciente. Tais meios são fundamentais na segurança, pois são verificadas todas as etapas do processo desde o planejamento até a entrega de dose levando em consideração todas as particularidades do tratamento $[6,9]$.

Para verificação específica da entrega da dose prescrita ao paciente, um dos métodos empregados na radioterapia é a medida da dose prescrita em um ponto determinado em um fantoma utilizando câmara de ionização e/ou filme radiocrômico para obtenção do valor da dose, fornecido pela câmara de ionização, juntamente com o plano de distribuição de dose e a dose fornecidos pelo filme radiocrômico [10]. Neste método, os resultados obtidos com os dois detectores são comparados com o sistema de planejamento para verificar a segurança do processo.

No caso da radiocirurgia, há grande dificuldade em realizar dosimetria em regiões com pequenos campos de irradiação (lesões menores que $0,5 \mathrm{~cm}^{3}$ ) e grande gradiente de dose (doses nas regiões adjacentes, até $0,5 \mathrm{~cm}$ do volume alvo, devem receber no máximo até $50 \%$ da dose prescrita), pois os medidores e detectores, tradicionalmente utilizados nos centros de radioterapia, como a câmara de ionização e filmes radiocrômicos, possuem restrições para esse tipo de medida. As câmaras de ionização e semicondutores, por exemplo, não fornecem a resolução espacial que alguns planejamentos necessitam, pois fornecem o valor de dose medido no seu volume não fornecendo a distribuição de dose num plano. Já os filmes radiocrômicos apresentam "efeito de borda" dificultando medidas em campos pequenos de irradiação [11].

Dentre os sistemas dosimétricos disponíveis, tem-se os baseados na luminescência opticamente estimulada (Optically Stimulated Luminescence - OSL). Devido à natureza óptica do processo, alguns detectores OSL, comerciais como o Thermalox 995 (Materion Co) de óxido de berílio e o Luxel (Landauer Inc) de óxido de alumínio, ou desenvolvidos nacionalmente, como as folhas OSL baseadas na fluorita natural brasileira, apresentam como principal vantagem a possibilidade de reavaliação das doses (releitura), avaliação da distribuição e perfil de dose [12-14].

Considerando a necessidade de meios precisos e eficientes para dosimetria em campos pequenos e as vantagens dos detectores OSL, neste trabalho foi comparado o controle de qualidade feito com 
câmara de ionização durante o planejamento de tratamentos de radiocirurgia (padrão ouro) com o controle de qualidade empregando folhas detetoras OSL de fluoreto de cálcio $\left(\mathrm{CaF}_{2}\right)$ baseadas na fluorita nacional brasileira.

\section{MATERIAIS E MÉTODOS}

Para comparar os controles de qualidade utilizando câmara de ionização e as folhas detectoras, foram realizados três planejamentos no sistema o MonacoElekta com fracionamento único (doses de 300 cGy, 600 cGy e 2100 cGy) utiizando a tecnologia AVM e empregando fótons com energia de $6 \mathrm{MV}$. Este foi feito para um adulto padrão utilizando a tomografia do simulador antropomórfico adulto Alderson Radiation Therapy Phanton (ART 210), como mostrado na Figura 1, simulando uma lesão menor do que $0,5 \mathrm{~cm}^{3}$.

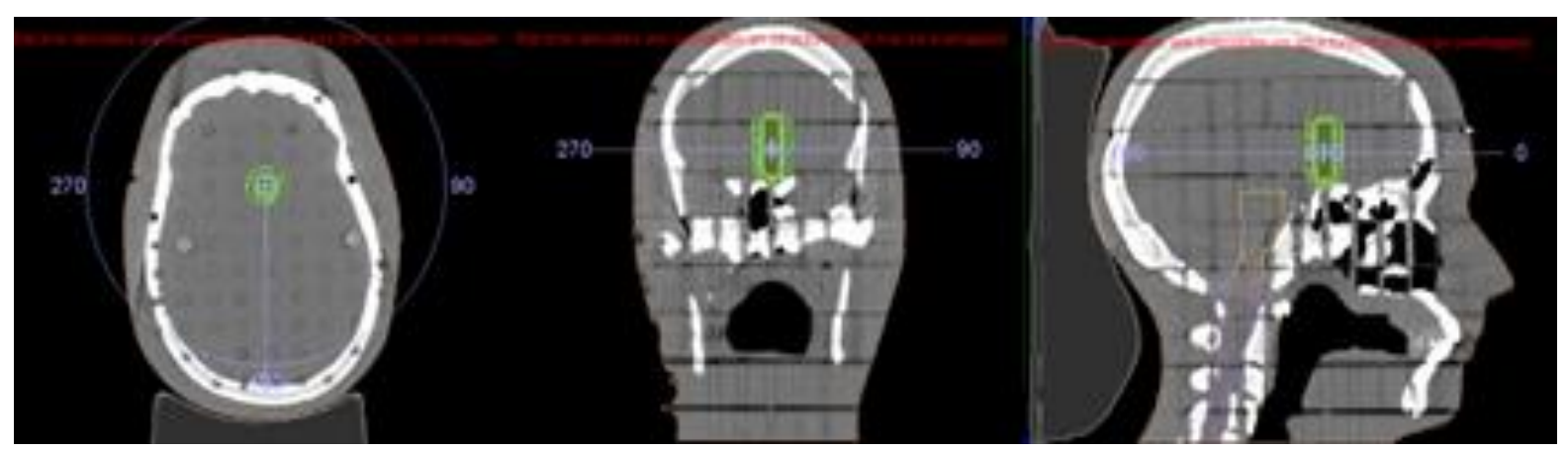

Figura 1: Planejamento da radiocirurgia (AVM) em simulador antropomórfico.

Para criar o controle de qualidade do tratamento proposto utilizando o MonacoElekta, o planejamento completo realizado no simulador antropomórfico (mesmo arco/ângulo de gantry, mesmos segmentos e as mesmas unidades monitoras e suas respctivas doses de tratamento) foi transferido à tomografia de um fantoma, composto por placas de polimetilmetacrilato com densidade equivalente à água popularmente conhecidas como placas de "água sólida", com encaixe para câmara de ionização em região central, medindo $30 \times 30 \mathrm{~cm}^{2}$ totalizando 16 placas de $1 \mathrm{~cm}$ de espessura, 2 placas de $0,5 \mathrm{~cm}$ formando assim um fantoma de dimensões $30 \mathrm{x} 30 \times 17 \mathrm{~cm}^{3}$. Vale ressaltar que este fantoma atende as recomendações do Report TG 119, no qual sugere-se a realização de medições, utilizando fantomas, preservando todos os parâmetros apresentados no 
planejamento e o uso de câmaras de ionização com volumes sensíveis inferiores a $0,6 \mathrm{~cm}^{3}$ como as semi-flex $\left(0,125 \mathrm{~cm}^{3}\right)$ ou as câmaras de ionização Pinpoint $\left(0,016 \mathrm{~cm}^{3}\right)$, pois câmaras de ionização com volumes maiores são mais susceptíveis a variações da distribuição de dose no volume analisado [15].

A Figura 2 mostra o fantoma de polimetacrilato e o encaixe da câmera de ionização PTW semiflex (modelo TN31010) utilizada para medida de dose no fantoma.

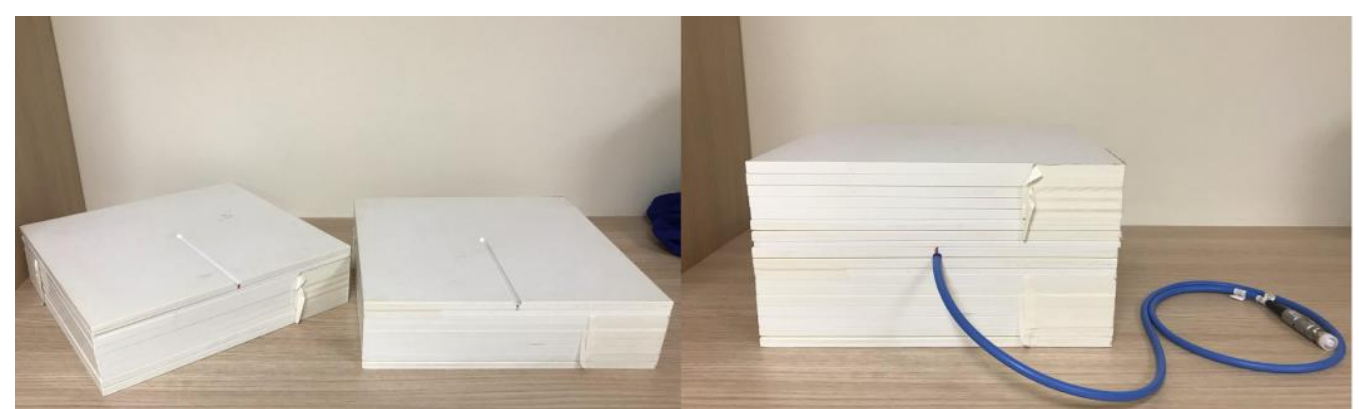

Figura 2: Fantoma de polimetilmetacrilato de $30 \times 30 \times 17 \mathrm{~cm}^{3}$, com densidade equivalente à água, composto por placas de $1 \mathrm{~cm}$, com encaixe para câmera de ionização ( a esquerda) e câmara de ionização PTW semi-flex modelo TN31010 inserida no fantoma.

$\mathrm{O}$ isocentro de tratamento foi posicionado no fantoma, no sistema de planejamento MonacoElekta, para que o volume sensível da câmera de ionização ficasse na região homegênea de dose (região do volume-alvo de maior dose) diminuindo as incertazas durante as medições, conforme mostrado na Figura 3.

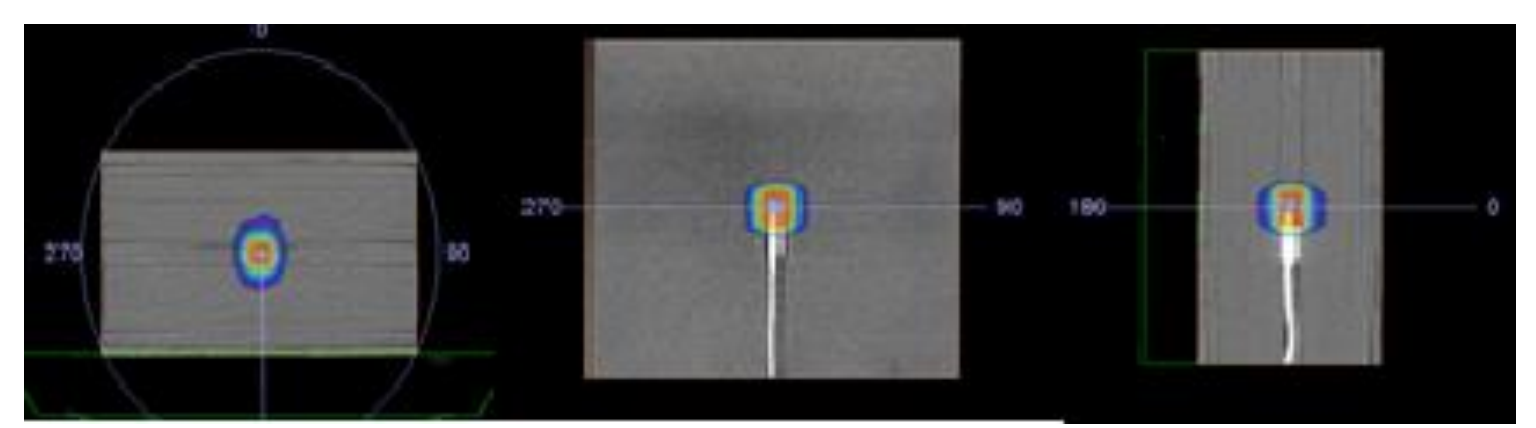

Figura 3: Fantoma polimetilmetacrilato $30 \times 30 \times 17 \mathrm{~cm}^{3} \mathrm{com}$ densidade equivalente à água no sistema de planejamento Monaco Elekta. 
As irradiações para controle de qualidade foram realizadas utilizando um acelerador linear Elekta Synergy Full com feixe de fótons de 6 MV, empregando a câmara de ionização PTW semiflex, acoplada a um eletrômetro PTW (Unidos E) conforme a Figura 4 , posicionada no centro do fantoma (placas $30 \times 30 \mathrm{~cm}^{2}$ com densidade equivalente à água totalizando 17 centímetros de altura), conforme já realizado na rotina da clínica para validação da entrega da dose planejada. A exposição indicada pela câmara de ionização medida em um único ponto, foi convertida em dose e comparada àquela prevista pelo sistema de planejamento (dose prescrita).

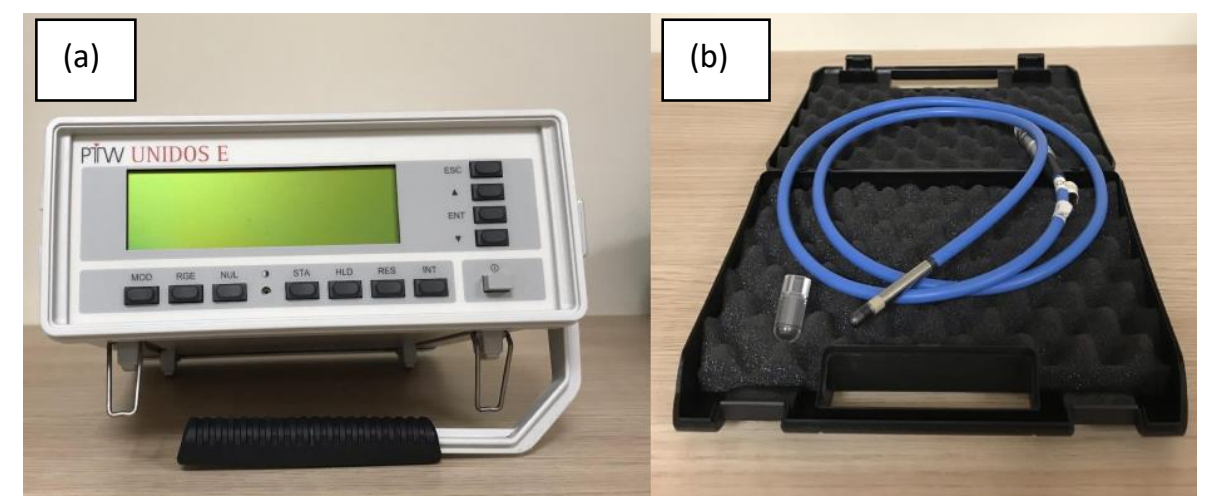

Figura 4: (a) Eletrômetro PTW (Unidos) e (b) Câmara de ionização PTW semi-flex (modelo TN31010).

As folhas detectoras OSL de $\mathrm{CaF}_{2}$ foram produzidas a partir da fluorita nacional brasileira utilizando à técnica a frio desenvolvida na Universidade Tecnológica Federal do Paraná em Curitiba. A Figura 5 mostra as folhas detectoras OSL, em diferentes formatos, que foram cortadas em diferentes tamanhos para o controle de qualidade, e embaladas em papel cartão preto, quando necessário, para prevenir a exposição a luz [14].

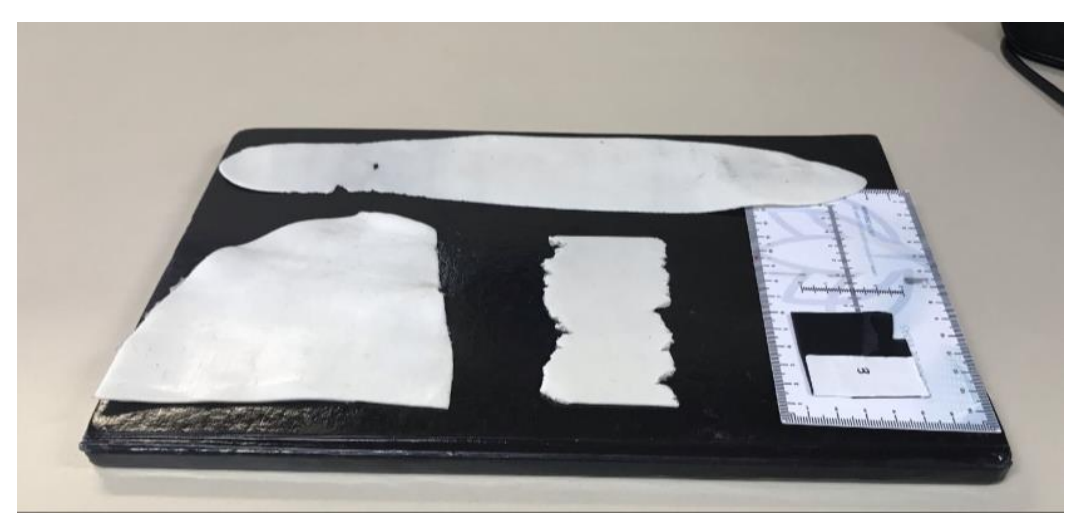

Figura 5: Folhas detectoras OSL de $\mathrm{CaF}_{2}$ produzidas na UTFPR-CT. 
Para o controle de qualidade empregando as folhas detectoras OSL de $\mathrm{CaF}_{2}$, estas foram calibradas para energia de fótons de $6 \mathrm{MV}$. Primeiramente, tiras de folhas detectoras OSL, com aproximadamente $2 \mathrm{~cm}$ de comprimento, $0,5 \mathrm{~cm}$ de largura e menores que dois milimetros de espessura, embaladas aos pares em plástico preto radiotransparente, foram irradiadas no acelerador linear com fótons de $6 \mathrm{MV}$ utilizando doses de 100 a 1000 cGy.

As leituras foram realizadas em um leitor OSL linear desenvolvido e em funcionamento no Instituto de Física da Universidade de São Paulo (IFUSP), mostrado na Figura 6. Este foi utilizado no modo pulsado empregando LED verdes para estimulação (aproximadamente $500 \mathrm{~nm}$ ). Para leitura das folhas detectoras, estas foram cortadas em tiras de aproximadamente $0,5 \mathrm{~cm}$ de largura. Neste leitor, os valores de intensidade OSL são integrados no intervalo de tempo entre os pulsos de estimulação (1000 ms) conforme a fita OSL é deslocada na plataforma do leitor a cada $1 \mathrm{~mm}$. Com isso foi avaliado o fator de calibração, utilizado para converter os valores de intensidade OSL (sinal luminoso) em dose (em cGy).

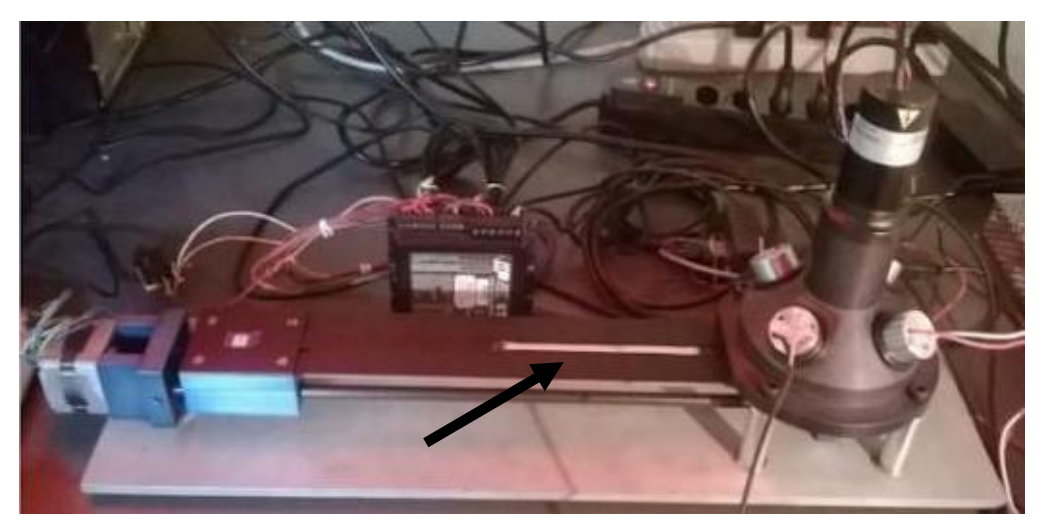

Figura 6: Leitor OSL linear do IFUSP e fita detectora OSL de $\mathrm{CaF}_{2}$ identificada (seta preta).

Para a validação da dose planejada a ser entregue utilizando as folhas OSL de $\mathrm{CaF}_{2}, 3$ fitas detectoras OSL, com as mesmas dimensões das tiras utilizadas na calibração e embaladas separadamente em plástico preto radiotransparente, foram posicionadas na mesma localização da câmara de ionização no fantoma composto por placas de polimetilmetacrilato (Figura 7). Em seguida, para verificar as doses de 300, 600 e 2100 cGy planejadas, foram realizadas as irradiações do mesmo modo que foi feito para verificar as doses com a câmara de ionização PTW. Assim como na calibração, as fitas foram lidas no leitor OSL linear no modo pulsado. 


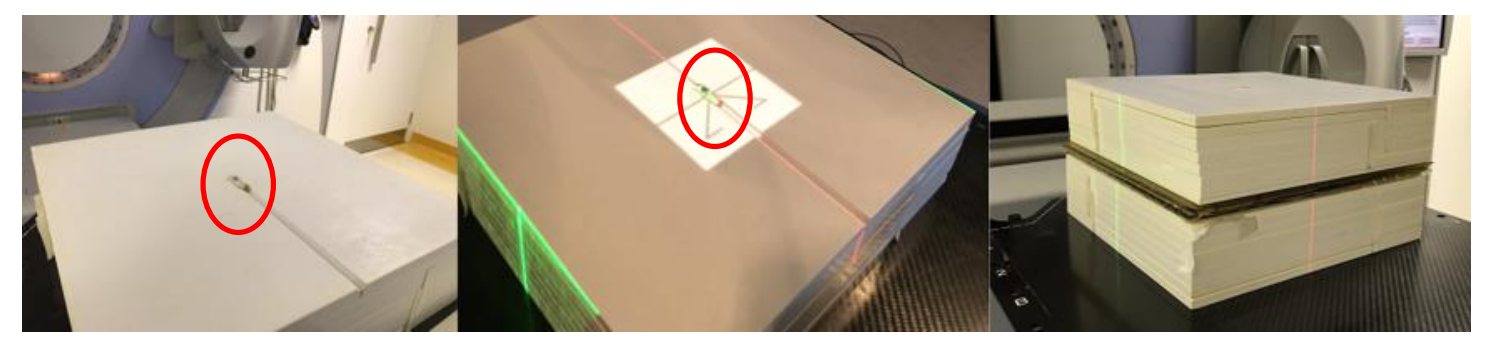

Figura 7: Posicionamento folha OSL de CaF $\mathrm{O}_{2}$ (identificada por círculos vermelhos) para validação da dose planejada.

\section{RESULTADOS E DISCUSSÃO}

A Tabela 1 apresenta os valores de dose de tratamento (prescrita), e o valor de dose máxima dado/encontrado no sistema de planejamento os valores medidos com a câmara de ionização (CI) e os valores de dose avaliados ao longo dos comprimentos das fitas OSL de $\mathrm{CaF}_{2}$. 
Tabela 1: Doses fornecidas através do sistema de planejamento (dose de tratamento/dose máxima), medições com $\mathrm{CI}$ e doses avaliadas com as fitas detectoras OSL de $\mathrm{CaF}_{2}$.

\begin{tabular}{ccc}
\hline \multicolumn{3}{c}{ Dose de tratamento/ dose máxima (cGy) } \\
\hline $300 / 428$ & $600 / 740$ & $2100 / 2320$ \\
\hline \multicolumn{3}{c}{ Dose CI (cGy) } \\
\hline 304 & 633 & 2040 \\
\hline \multicolumn{3}{c}{ Dose fita OSL de CaF $\mathbf{~ ( c G y ) ~}$} \\
\hline $287 \pm 23$ & $691 \pm 54$ & $2259 \pm 177$ \\
$282 \pm 22$ & $688 \pm 54$ & $2341 \pm 184$ \\
$282 \pm 22$ & $749 \pm 59$ & $2235 \pm 176$ \\
$311 \pm 24$ & $737 \pm 58$ & $1962 \pm 154$ \\
$292 \pm 23$ & $796 \pm 62$ & $2019 \pm 159$ \\
$307 \pm 24$ & $759 \pm 60$ & $2314 \pm 182$ \\
$302 \pm 24$ & $791 \pm 62$ & $2631 \pm 182$ \\
$332 \pm 26$ & $798 \pm 63$ & $2674 \pm 210$ \\
$359 \pm 28$ & $762 \pm 60$ & $2549 \pm 200$ \\
$380 \pm 30$ & $740 \pm 58$ & $2522 \pm 198$ \\
$358 \pm 28$ & $750 \pm 59$ & $2655 \pm 209$ \\
$285 \pm 22$ & $797 \pm 63$ & $2613 \pm 205$ \\
\hline
\end{tabular}

Comparando os valores de dose avaliados para cada um dos três planejamentos ao longo do comprimento das fitas detectoras OSL com os valores de dose fornecidos pelo equipamento medido com a câmara de ionização (CI), observamos que estes são compatíveis e que, conforme já previsto no planejamento, doses maiores (valores máximos) do que as doses de tratamento foram observadas ao longo do comprimento das tiras. Os valores máximos de dose dados nos planejamentos foram de 428, 740 e 2320 cGy e as doses máximas avaliadas no controle de qualidade com as folhas OSL de $\mathrm{CaF}_{2}$ foram de $(380 \pm 30)$, (798 \pm 63$)$ e $(2674 \pm 210) \mathrm{cGy}$. Vale ressaltar que para as duas doses mais baixas, os valores do planejamento e avaliados com as folhas estão dentro das incertezas e, para 
dose mais alta de tratamento, este está ligeiramente mais alto, porém não foram consideradas as incertezas associadas do planejamento.

Vale também destacar que embora as incertezas associadas aos valores estimados com as folhas OSL de $\mathrm{CaF}_{2}$ sejam de aproximadamente 8\%, estas são intrínsecas ao sistema dosimétrico na avaliação das contagens de fundo do detector e de escuro do leitor OSL empregado.

Observando os valores medidos com câmara de ionização, vemos que estes podem diferir do valor da dose de tratamento, isso porque no planejamento se tem uma distribuição de dose. Então, embora a câmara possa ser posicionada no ponto quente, onde se tem uma maior perspectiva de se medir o valor máximo, como se tem a dose em um volume relativamente grande, as doses medidas com a câmara estão entre o valor de tratamento e o valor máximo. Já com as folhas OSL no controle de qualidade, é possível observar o gradiente de dose na região de tratamento e com uma boa resolução espacial mesmo aplicando campos pequenos, como no caso da radiocirurgia utilizando AVM.

\section{CONCLUSÕES}

Neste trabalho foram feitos controles de qualidade para tratamentos de radiocirurgia utilizando a tecnologia AVM, na qual são empregados campos pequenos de irradiação, utilizando câmara de ionização, atualmente "padrão ouro" nesta modalidade e folhas detectoras OSL de $\mathrm{CaF}_{2}$. Os resultados mostram que tanto para doses baixas como para doses mais elevadas, é possível avaliar a distribuição de dose ao longo do volume pequeno irradiado sendo compatível com as distribuições de doses dadas no planejamento a partir da dose de tratamento. As folhas detectoras OSL de $\mathrm{CaF}_{2}$ apresentaram boa resolução espacial e resultados compatíveis com os valores medidos com a câmara de ionização, validando sua aplicação para campos pequenos e regiões com altos gradientes de dose.

Deste modo, concluímos neste estudo, que as folhas detectoras OSL de $\mathrm{CaF}_{2}$ podem ser aplicadas no controle de qualidade do planejamento individualizado dos pacientes submetidos a radiocirurgia com tecnologia AVM, com a vantagem de permitir a visualização da distribuição de dose, perfil e não apresentar limitações de eletrônica associada como é o caso das câmaras de ionização. 


\section{REFERÊNCIAS}

[1] WHO - World Health Organization. Radiotherapy Risk Profile. Technical Manual, Geneva 2008.

[2] MINTZ, AH et al. A randomized trial to assess the efficacy of surgery in addition to radiotherapy in patients with a single cerebral metastasis. Cancer, v. 78, p. 1470-1476, 1996.

[3] STUDENSKI, M.T. et al. Clinical experience transitioning from IMRT to VMAT for head and neck cancer. Medical Dosimetry 38, p. 171-175, EUA, 2013.

[4] ICRU - International Commission on Radiation Units and Measurements. Prescribing, recording, and reporting photon beam therapy. ICRU Report 50, Bethesda: ICRU, 1993.

[5] PODGORSAK, E. B. Radiation oncology physics: a handbook for teachers and students. International Atomic Energy Agency. IAEA. Áustria, 2005.

[6] CNEN - Comissão Nacional de Energia Nuclear. Requisitos de segurança e Proteção Radiológia para Serviços de Radioterapia. CNEN NN 6.10 (atualizada), Rio de Janeiro, 2017.

[7] IAEA - International Atomic Energy Agency. Absorbed dose determination in external beam radiotherapy: An international code of practice for dosimetry based on standards of absorbed dose to water. Technical Report Series, IAEA TRS-398, Vienna, 2000.

[8] INCA - Instituto Nacional do Cancer José de Alencar Gomes da Silva. Ministério da Saúde. TEC DOC 1151 (tradução) aspectos físicos da garantia da qualidade em radioterapia, IAEA TEC DOC 1151, Rio de janeiro, 2000.

[9] AGAZARYAN, N.; SOLBERG, TD.; DEMARCO, JJ. Patient specific quality assurance for the delivery of intensity modulated radiotherapy. J Appl Clin Med Phys, p 40-50, 2003.

[10] ESTRO - European Society for Therapeutic Radiology and Oncology. Guidelines for the verification of IMRT. Brussels, 2008. 
[11] NIROOMAND et al. Radiochromic film dosimetry. Recommendations of AAPM Radiation Therapy Committee Task Group 55, Medical Physics, vol. 25, No. 11, 1998.

[12] JAHN, A.; SOMMER, M.; HENNIGER, J. 2D - OSL - Dosimetry with beryllium oxide. Germany, 2010.

[13] YUKIHARA, E. G. et al. An optically stimulated luminescence system to measure dose profiles in x-ray computed tomography, EUA, 2009.

[14] MALTHEZ, A. L. M. C., el al. Low-cost flexible OSL detector leaf for 2D radiation measurements. ISPR14, Cordoba, 2018.

[15] EZZEL G. A., BURMEISTER J. W., DOGAN N., et al. IMRT Commissioning: Multiple Institution Planning and Dosimetry Comparisons, a Report from AAPM Task Group 119. Medical Physics, 2009. 